\title{
两种性比类群的雄性黄山短尾猴繁殖行为和攻击行为比较
}

\author{
王 希, 李进华*，夏东坡，陈 燃，朱勇，张 敏，王 粟 \\ (安徽大学 生命科学学院, 安徽省生态工程与生物技术重点实验室, 安徽省生态经济技术研究中心, 安徽 合肥 230039)
}

摘要: 有效性比 (operational sex ratio, OSR) 是指性成熟雄性数量与发情雌性数量的比值, 可作为测量 性选择强度的指标。本文对两种有效性比的黄山短尾猴(Macaca thibetana) 鱼鳞坑 $\mathrm{YA}_{1}$ 群和YA 2 群成年雄性在交 配期（2007 年 8-12 月）内的繁殖行为和攻击行为进行研究, 采用目标动物取样法、随机取样法和连续记录 法记录行为, 探讨有效性比对雄性黄山短尾猴交配竞争的影响。研究期间, $\mathrm{YA}_{1}$ 群的有效性比为 0.4:1, YA $\mathrm{Y}_{2}$ 群 的有效性比为 0.9:1, $\mathrm{YA}_{2}$ 群的有效性比大于 $\mathrm{YA}_{1}$ 群。在繁殖行为上, 高顺位成年雄性的性检查、做鬼脸、性 追赶和交配行为在两群间均存在显著差异, $\mathrm{YA}_{1}$ 群高于 $\mathrm{YA}_{2}$ 群 $(P<0.01)$; 中等顺位成年雄性的性检查、做鬼 脸、性追赶在两群间均存在显著差异, $\mathrm{YA}_{1}$ 群高于 $\mathrm{YA}_{2}$ 群 $(P<0.01)$, 交配行为在两群间存在显著差异, $\mathrm{YA}_{1}$ 群 高于YA 2 群 $(P<0.05)$; 低顺位成年雄性在两群间不存在显著差异。在攻击行为上, 高顺位成年雄性在两群间 存在显著差异, $\mathrm{YA}_{2}$ 群高于 $\mathrm{YA}_{1}$ 群 $(P<0.01)$; 中等顺位成年雄性在两群间存在显著差异, $\mathrm{YA}_{2}$ 群高于 $\mathrm{YA}_{1}$ 群 $(P$ $<0.05)$; $\mathrm{YA}_{1}$ 群低顺位成年雄性攻击行为发生频次为零。结果表明, 雄雌有效性比越大, 雄性黄山短尾猴的 交配机会越少, 繁殖行为发生频次下降, 竞争压力增大, 攻击行为频次上升, 因此 $\mathrm{YA}_{2}$ 群成年雄性交配竞争 强度高于YA 1 群。本研究结果支持性选择理论中有效性比对交配竞争作用的预测。

关键词: 短尾猴; 有效性比; 繁殖行为; 攻击行为; 性选择理论

中图分类号: Q959.848 文献标识码: A 文章编号：0254-5853-(2009)01-0083-07

\section{Comparisons on Reproductive and Aggressive Behaviors in Male Tibetan Macaques (Macaca thibetana) of Two Different Operational Sex Ratio Troops at Huangshan, China}

\author{
WANG Xi, LI Jin-hua ${ }^{*}$, XIA Dong-po, CHEN Ran, ZHU Yong, ZHANG Min, WANG Su \\ (School of Life Science, Anhui University; Anhui Key Laboratory of Eco-engineering and Bio-technique; Anhui Research \\ Center of Ecological Economy, Hefei 230039, China)
}

\begin{abstract}
Operational sex ratio (OSR) is the ratio of the number of sexually mature males to the estrous females, which is used to predict the intensity of sexual selection. During the mating season (August-December 2007), we studied reproductive and aggressive behaviors in sexually mature male Tibetan macaques (Macaca thibitana) in two troops ( $\mathrm{YA}_{1}$ and $\left.\mathrm{YA}_{2}\right)$ at Huangshan, China. The behavioral data were collected by focal animal sampling, ad libitum sampling and continuous recording. The purpose is to discuss the impact of OSR on mating competition of male Tibetan macaques. The OSR of $\mathrm{YA}_{1}$ was 0.4:1, and the OSR of $\mathrm{YA}_{2}$ was 0.9:1. The male-biased in OSR of $\mathrm{YA}_{2}$ was higher than $\mathrm{YA}_{1}$. The sexually mature males of high rank in $\mathrm{YA}_{1}$ troop showed higher rates of reproductive behaviors than the males in $\mathrm{YA}_{2}$ : genital-inspecting, grimace, sexual chase and mating, respectively $(P<0.01)$. The sexually mature males of middle rank in $\mathrm{YA}_{1}$ troop showed higher rates of reproductive behaviors than the males in $\mathrm{YA}_{2}$ : genital-inspecting, grimace, sexual chase $(P<0.01)$. The sexually mature males of middle rank in $\mathrm{YA}_{1}$ troop also showed higher rate of mating behavior than the males in $\mathrm{YA}_{2}(P<0.05)$. However, there was no significant difference in reproductive behaviors between sexually mature males of low rank in two troops. The sexually mature males of high rank in $\mathrm{YA}_{2}$ troop showed higher rate of aggressive behavior than the males in $\mathrm{YA}_{1}(P<0.01)$. The sexually mature males of middle rank in $\mathrm{YA}_{2}$ troop showed higher rate of aggressive behavior than the males in $\mathrm{YA}_{1}(P<0.05)$. The sexually mature males of low rank in $\mathrm{YA}_{1}$ troop didn't show any
\end{abstract}

收稿日期：2008-09-28; 接受日期: 2008-12-4

基金项目: 国家自然科学基金(30570237, 30770288); 安徽省教育厅科技创新团队(TD200703); 安徽省人才开发基金; 安徽大学学术创新团 队项目基金资助

*通讯作者（Corresponding author), E-mail: jhli@ahu.edu.cn 
aggressive behavior. These results suggested that the intensification of male-biased in OSR can lead to the decrease of reproductive behaviors and the increase of aggressive behavior of male Tibetan macaques. The intensity of mating competition of sexually mature males in $\mathrm{YA}_{2}$ troop was stronger than the males in $\mathrm{YA}_{1}$. Our results support the prediction of the impact of OSR on mating competition by sexual selection theory.

Key words: Tibetan macaques (Macaca thibetana); Operational sex ratio; Reproductive behavior; Aggressive behavior; Sexual selection theory

有效性比（operational sex ratio, OSR）是指性 成熟雄性数量与发情雌性数量的比值。性选择理论 (sexual selection theory) 预测有效性比越大, 意味 着成年雄性繁殖竞争对手越多, 压力越大, 交配机 会越少, 雄性之间的交配竞争越激烈 (Mitani \& John, 1990 )。有效性比是影响雄性间交配竞争强度的一 个重要因素 (Gwynne, 1991; Clutton-Brock \& Parker, 1992), 可作为测量性选择强度的指标 (Emlen \& Oring, 1977)。

性选择理论中关于有效性比对交配竞争作用 的预测提出后, 大部分验证研究集中在鱼类 (Quinn et al, 1996; Grant et al, 1995)、爬行类 (Patrick \& Jean-Francois, 2008) 和岛类 (Mark \& Lewis, 1988)。 在欧洲鳑鲏 (Rhodeus sericeus) (Martin et al, 2004) 和沙䰃虾虎鱼 (Pomatoschistus minutes) (Kvarnemo et al, 1995）中, 雄性的攻击行为发生频次随着雄雌 有效性比的增大而升高。有效性比越向雄性一方偏 离, 交配竞争越加激烈, 欧洲龙虾 (Homarus gammarus) (Valerie et al, 1999) 和果子狸 (Paguma larvata）（Jia et al, 2000）的雄性之间会互相干扰对 方的交配, 导致交配行为发生频次降低。在古比鱼 (Poecilia reticulata) 中, 当有效性比越大时, 不仅 使雄性交配频次减少, 而且也会影响古比鱼繁殖行 为的方式, 即雄性数量增多时, 雄性古比鱼将减少 在雌性面前的“S”型展示 (Jirotkul, 1999)。

已有研究表明有效性比对非人灵长类的交配 竞争也有影响（Bulger, 1993; Paul, 1997)。例如, 雄雌有效性比的增大会使一些低顺位雄性日本猴 (Macaca fuscata fuscata) 个体很难得到交配机会, 导致交配竞争加剧（Takahashi, 2001；2004); 雄性 草原狒狒 (Papio cynocephalus) 个体的交配机会随 着雄雌有效性比中能够交配雌性数量的下降而减 少 (Fred, 1995); 相反, 在北非短尾猴 (Macaca sylvanus) 中, 发情雌性数量增加, 即当有效性比 减小时, 使得交配竞争压力减小, 雄性交配次数增 多 (Michael et al, 2007)。
然而, 繁殖行为和攻击行为受到多种因素的影 响和制约, 并非由单一的有效性比因素决定。例如, 雄性草原狒狒 (Papio cynocephalus) 射精交配的机 会与有效性比无关 (Fred, 1995); 在雄性花尾汤鲤 (Jordanella floridae) 中, 有效性比的变化对雄性 之间的攻击行为没有显著影响（Klug et al, 2008); 有效性比对低顺位雄性布氏田鼠 (Microtus brandti) 的社会行为基本没有影响（Zhang \& Shi, 2006)。因 此, 有效性比对于繁殖行为和攻击行为的影响有待 于进一步研究。此外, 竞争是动物行为进化的动力 (Sun, 2001), 雄性偏多导致的雄性间竞争加剧, 能够影响雄性的交配策略和繁殖成功（Jirotkul, 1999)。因此通过对不同有效性比下交配竞争程度 的研究既可以验证性选择理论中有效性比对交配 竞争作用的预测, 又可以分析交配竞争对于雄性交 配策略和繁殖成功及其行为的影响。

黄山短尾猴 (Macaca thibetana) 是多雄多雌的 自然社会群体 ( Li et al, 2007), 交配期为 7 至 12 月, 短尾猴的交配竞争主要发生在这个时期 $(\mathrm{Li}$, 1999 )。研究群鱼鳞坑YA 1 群和YA 2 群的有效性比

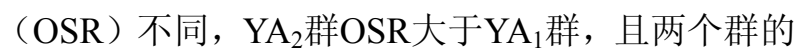
OSR 在研究期间没有变化, 这为验证有效性比对交 配竞争作用的预测提供了难得的条件。繁殖行为和 攻击行为是交配竞争的行为体现, 根据性选择理论 中有效性比对交配竞争作用的预测, 本研究假设: $\mathrm{YA}_{1}$ 群和 $\mathrm{YA}_{2}$ 群繁殖行为和攻击行为发生频次存在 差异, $\mathrm{YA}_{2}$ 群成年雄性交配竞争强度大于YA1 群。

\section{1 材料和方法}

\section{1 研究地点与研究对象}

研究群为栖息于黄山风景区西南山麓的鱼鳞

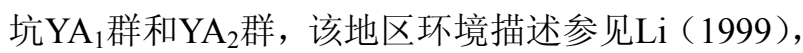
本课题组自 1986 年以来对YA 1 群进行连续 20 年的 观察研究, $\mathrm{YA}_{2}$ 群于 1996 年从YA 1 群分群出来 ( $\mathrm{Li}$ et al, 1996), 两群间以一条小溪为界, 有各自的领域, 在观察期内两群之间没有直接交流。两群均进行全 
年人工定时定点定量投喂, 每天在喂食场各投喂 4 次玉米, 但猴群的主要食物仍然均是天然的野生植 物, 两个猴群除了在喂食场受食, 大部分时间在森 林中活动。

本研究中共观察 $\mathrm{YA}_{1}$ 群 3 个成年雄性个体, $\mathrm{YA}_{2}$ 群 10 个成年雄性个体。通过攻击一屈服行为指标, 确定同一群体内不同个体的等级关系 ( $\mathrm{Li}, 1999$; Chen et al, 2008)。在观察期内用随机取样法 ( Ad lib sampling）分别记录YA 1 群 3 个个体攻击一屈服回合
35 次, $\mathrm{YA}_{2}$ 群 10 个个体攻击一屈服回合 201 次 (Altmann, 1974)。为了便于在两群间横向比较相 同等级成年雄性的行为差异, 我们依据攻击一屈服 行为和成年雄性的临时配偶情况，将研究对象划分 为 3 个社会等级: 高顺位、中等顺位和低顺位（表 1 和表 2)。

本研究在交配期内对 13 只目标个体观察 153 天 (2007 年 8-12 月), 目标动物取样时间为 $241 \mathrm{~h}$

表 1 黄山风景区西南山麓YA群研究个体的社会等级结构

Tab. 1 Dominance hierarchy among the study individuals of $\mathrm{YA}_{1}$ troop in southwest of Huangshan scenic spot

\begin{tabular}{lcccc}
\hline & \multicolumn{3}{c}{ 劣位者 Subordinates } \\
\cline { 3 - 5 } & & 高顺位 High Rank & 中等顺位 Middle Rank & 低顺位 Low Rank \\
\cline { 3 - 5 } & 子龙 ZL & 高山 GS & 黄盖 HG \\
\hline 优位者 & 子龙 ZL & 9 & 16 \\
Dominants & 高山 GS & & 10 \\
& 黄盖 HG & & \\
\hline
\end{tabular}

表 2 黄山风景区西南山麓Y群研究个体的社会等级结构

Tab. 2 Dominance hierarchy among the study individuals of $\mathrm{YA}_{2}$ troop in southwest of Huangshan scenic spot

\begin{tabular}{|c|c|c|c|c|c|c|c|c|c|c|}
\hline \multirow{4}{*}{$\begin{array}{c}\text { 优位者 } \\
\text { Dominants }\end{array}$} & \multicolumn{10}{|c|}{ 劣位者 Subordinates } \\
\hline & \multicolumn{3}{|c|}{ 高顺位 High Rank } & \multicolumn{3}{|c|}{ 中等顺位 Middle Rank } & \multicolumn{4}{|c|}{ 低顺位 Low Rank } \\
\hline & 丑八怪 & 白脸 & 高鼻子 & 长筒 & 九天 & 独蛋 & 三雄 & 叶贵 & 叶天 & 花郎 \\
\hline & CBG & $\mathrm{BL}$ & GBZ & $\mathrm{CT}$ & $\mathrm{JT}$ & DD & $\mathrm{SX}$ & YG & YT & $\mathrm{HL}$ \\
\hline 丑八怪 $\mathrm{CBG}$ & & 5 & 7 & 2 & 10 & 3 & 8 & 12 & 5 & 3 \\
\hline 白脸 BL & & & 3 & 4 & 6 & 1 & 4 & 7 & 5 & 2 \\
\hline 高鼻子 GBZ & & & & 3 & 7 & 3 & 1 & 9 & 2 & 5 \\
\hline 长筒 $\mathrm{CT}$ & & & & & 5 & 1 & 3 & 6 & 3 & 1 \\
\hline 九天 JT & & & & & & 1 & 5 & 12 & 4 & 7 \\
\hline 独蛋 DD & & & & & & & 2 & 1 & 6 & 1 \\
\hline 三雄 $\mathrm{SX}$ & & & & & & & & 3 & 1 & 5 \\
\hline 叶贵 YG & & & & & & & & & 8 & 3 \\
\hline 叶天 YT & & & & & & & & & & 6 \\
\hline 花郎 HL & & & & & & & & & & \\
\hline
\end{tabular}

表 3 研究期间黄山风景区西南山麓YA群和YA群目标动物取样时间

Tab. 3 Duration for focal animal sampling of $\mathrm{YA}_{1} / \mathrm{YA}_{2}$ troop during studying period in southwest of Huangshan scenic spot

\begin{tabular}{|c|c|c|c|c|c|}
\hline \multicolumn{3}{|c|}{$\mathrm{YA}_{1}$ 群 $\mathrm{YA}_{1}$ troop } & \multicolumn{3}{|c|}{$\mathrm{YA}_{2}$ 群 $\mathrm{YA}_{2}$ troop } \\
\hline 雄性 Male & 取样时间 & Duration for sampling (min) & 雄性 Male & 取样时间 & Duration for sampling (min) \\
\hline \multirow{2}{*}{ 子龙（ZL） } & \multirow{2}{*}{\multicolumn{2}{|c|}{1120}} & 丑八怪 (CBG) & & 1120 \\
\hline & & & 白脸（BL） & & 1100 \\
\hline \multirow{4}{*}{ 高山（GS） } & \multirow{4}{*}{\multicolumn{2}{|c|}{1120}} & 高鼻子 (GBZ) & & 1100 \\
\hline & & & 长筒 (CT) & & 1100 \\
\hline & & & 九天 $(\mathrm{JT})$ & & 1120 \\
\hline & & & 独蛋（DD） & & 1100 \\
\hline \multirow{4}{*}{ 黄盖（HG） } & \multirow{4}{*}{\multicolumn{2}{|c|}{1120}} & 三雄 (SX) & & 1120 \\
\hline & & & 叶贵 $(Y G)$ & & 1100 \\
\hline & & & 叶天 (YT) & & 1120 \\
\hline & & & 花郎（HL） & & 1120 \\
\hline
\end{tabular}

括号内为研究对象的名称缩写。Letters in parentheses are the abbreviation for the study individuals' names. 
（Mean $\pm S D=18.54 \pm 0.17 \mathrm{~h}, n=13 ） （$ 表 3）。

\section{2 有效性比}

本研究中有效性比 (OSR) 是在整个交配期内, 成年雄性数量和潜在发情雌性数量的比值, 群体内 7 周岁以上的雄性为成年雄性（Li et al, 1995), 由 于雌性黄山短尾猴倾向于隐蔽自己的排卵, 同时缺 乏典型的性要求行为——发情, 雄性能够区别潜在 繁殖和不繁殖的雌性, 但不能对同一雌性的生理状 态做出准确判断 ( Li et al, 2005), 因此作者根据交 配期内参与交配的雌性情况、雌性性皮的颜色、雌 性交配的接受性以及雌性在产仔期的产仔情况, 来 判断雌性的潜在发情状况（Takahashi, 2002)。

\section{3 研究方法及行为定义}

采用目标动物取样法 (focal animal sampling) 和连续记录法 (continuous recording) 进行观察和 记录 (Altmann, 1974)。在观察前, 目标动物的观 察顺序通过抽签的方法进行随机选择, 取样时间 20 $\min$ 。根据行为定义, 用录音笔详细记录目标动物 个体在取样时间内发生的所有社会行为, 当找不到 目标个体或目标个体在观察时间内突然消失时, 按 观察顺序对下一个目标个体进行观察, 当先前消失 的目标个体出现时, 继续对其观察 (Li et al, 2005; Zhu et al, 2008)。记录的行为见表 4。

\section{4 数据处理和分析}

本研究结果中的行为数据来源于目标动物取 样法, 记录的行为用频次 (次/小时) 统计。利用SPSS 13.0 for Windows 统计软件进行分析 (Norusis, 2005 ), 数据类型为离散型, 故采用 $\chi^{2}$ Test分别分析 两群间相同等级成年雄性行为变量平均值的差异 显著性。显著性水平设为 $\alpha=0.05$ 。

\section{2 结 果}

\section{1 有效性比}

在交配期内, 两个群体的有效性比（OSR）没 有变化, $\mathrm{YA}_{1}$ 群的OSR为 $0.4: 1(3: 8), \mathrm{YA}_{2}$ 群的OSR



\section{2 繁殖行为}

（1）性检查行为: $Y_{1}$ 群高顺位成年雄性的性 检查行为发生频次显著高于 $\mathrm{YA}_{2}$ 群高顺位成年雄性 $\left(\chi^{2}=9.921, d f=1, P<0.01\right) ; \mathrm{YA}_{1}$ 群中等顺位成年 雄性的性检查行为发生频次显著高于YA 2 群中等顺 位成年雄性 $\left(\chi^{2}=17.065, d f=1, P<0.01\right)$; 低顺位 成年雄性的性检查行为发生频次在两群间无显著 差异 (图 1a)。

(2) 做鬼脸行为: $Y_{1}$ 群高顺位成年雄性的做 鬼脸行为发生频次显著高于YA 2 群高顺位成年雄性

表 4 行为定义

Tab. 4 Behavioral definitions

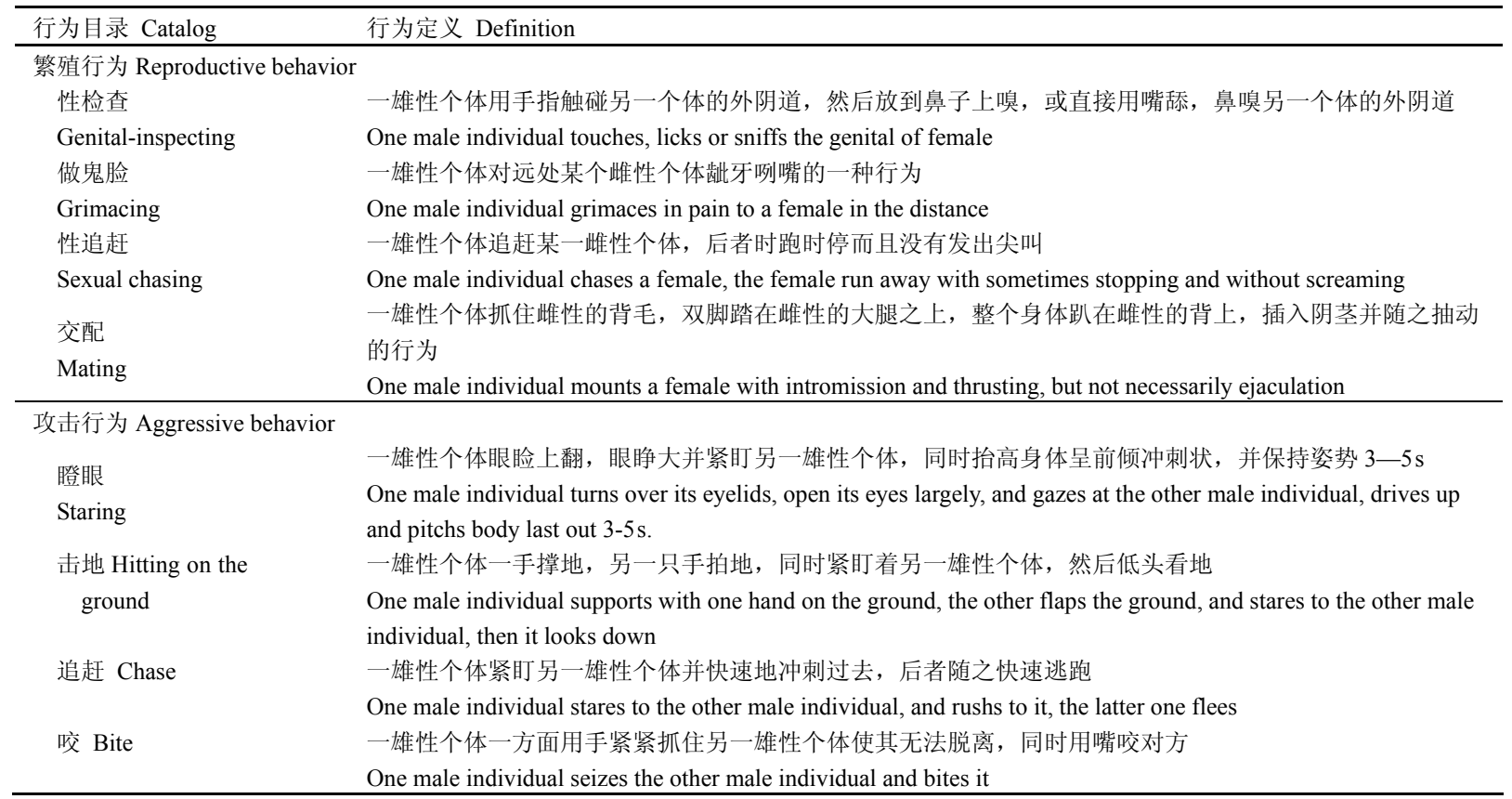

所以行为定义均参照 $\mathrm{Li}$ (1999)。本研究记录的攻击行为仅发生在成年雄性之间。

Behavior definition were referred to $\mathrm{Li}$ (1999). In this study, aggressive behavior only occurs in adult male individuals. 
$\left(\chi^{2}=9.323, d f=1, P<0.01\right) ; \mathrm{YA}_{1}$ 群中等顺位成年 雄性的做鬼脸行为发生频次显著高于 $Y_{2}$ 群中等顺 位成年雄性 $\left(\chi^{2}=9.000, d f=1, P<0.01\right)$; 低顺位 成年雄性的做鬼脸行为发生频次在两群间无显著 差异 (图 1b)。

（3）性追赶行为：YA 群高顺位成年雄性的性 追赶行为发生频次显著高于YA群高顺位成年雄性 $\left(\chi^{2}=11.571, d f=1, P<0.01\right) ; \mathrm{YA}_{1}$ 群中等顺位成年 雄性的性追赶行为发生频次显著高于YA 2 群中等顺 位成年雄性 $\left(\chi^{2}=8.333, d f=1, P<0.01\right)$; 低顺位成

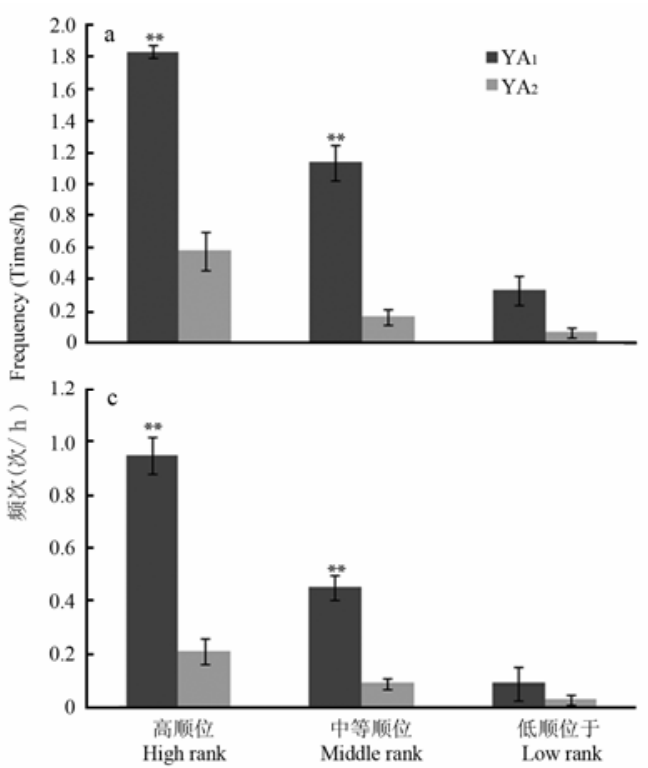

图 $1 \mathrm{YA}_{1}$ 群和 $\mathrm{YA}_{2}$ 群繁殖行为频次差异

Fig. 1 The reproductive behavior frequency between $\mathrm{YA}_{1}$ and $\mathrm{YA}_{2}$ troops

a: 性检查; b: 做鬼脸; c: 性追赶; d: 交配行为。

a: Genital-inspecting; b: Grimace frequency; c: Sexual chase frequency; d: Mating.

${ }^{*} P<0.05,{ }^{* *} P<0.01\left(\chi^{2}\right.$ Test $)$

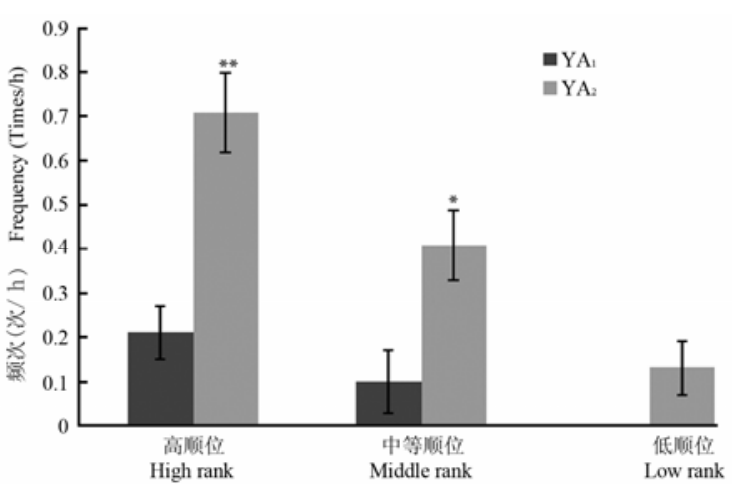

图 $2 \mathrm{YA}_{1}$ 群与 $\mathrm{YA}_{2}$ 群攻击行为频次差异

Fig. 2 The aggressive behavior frequency between $\mathrm{YA}_{1}$ and $\mathrm{YA}_{2}$ troops ${ }^{*} P<0.05,{ }^{* *} P<0.01\left(\chi^{2}\right.$ Test $)$.

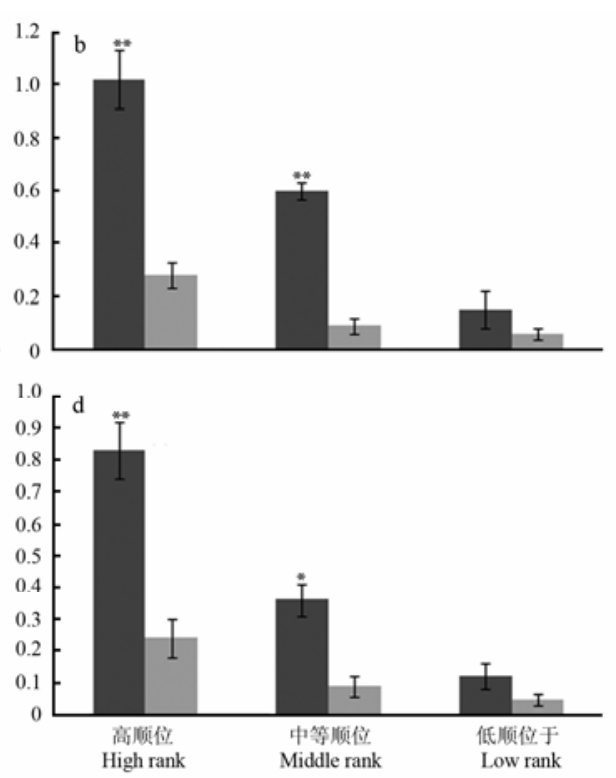

年雄性的性追赶行为发生频次在两群间无显著差 异 (图 1c)。

（4）交配行为: $\mathrm{YA}_{1}$ 群高顺位成年雄性的交配 行为发生频次显著高于 $\mathrm{YA}_{2}$ 群高顺位成年雄性 $\left(\chi^{2}=7.538, d f=1, P<0.01\right)$; $\mathrm{YA}_{1}$ 群中等顺位成年 雄性的交配行为发生频次显著高于YA群中等顺位 成年雄性 $\left(\chi^{2}=4.455, d f=1, P<0.05\right)$; 低顺位成年 雄性的交配行为发生频次在两群间无显著差异（图 1d)。

\section{3 攻击行为}


发生频次显著高于YA 1 群相对应顺位成年雄性, $\mathrm{YA}_{2}$ 群成年雄性间由于竞争导致的紧张程度要明显高 于YA 群。这些结果表明有效性比越大, 群体内成 年雄性个体数量越多, 雄性的交配竞争程度加剧, 使雄性间的攻击行为发生频次增加, 繁殖行为发生 频次降低, 交配机会减少。这与对日本狝猴

(Takahashi, 2004) 等的研究结果一致。本研究结 果验证了我们关于两个群体成年雄性交配竞争程 度差异性的假设, 支持了性选择理论中有效性比对 交配竞争作用的预测。

$\mathrm{YA}_{1}$ 群和YA 2 群成年雄性繁殖行为和攻击行为 频次差异的原因可能是由于高的对抗性是动物群 体内等级关系的一种表现, 对抗行为中优势一方比 弱势一方具有较高的等级地位, 从而可能获得更多 的资源、更好的保护巢区、寻找与多个异性个体交 配的机会，增加自身适合度 (Newsome, 1969; Baker, 1981 ), 高的对抗性也许是雄性保证繁殖成功的一 种交配策略。因此, 当有效性比中成年雄性增多时, 交配机会减少, 雄性为了得到高的繁殖成功, 对抗 性势必增大，攻击性随之上升。

不同有效性比导致两个群体内成年雄性表现 出不同程度的行为反应, 特别是两个群成年雄性攻 击行为发生频次的不同, 即 $\mathrm{YA}_{2}$ 群成年雄性的攻击 性水平高于YA 1 群, 攻击性的上升势必会造成雄性 间的关系更为紧张, 社群不稳定性增加, 这可能解

\section{参考文献:}

Altmann J. 1974. Observational study of behavior: sampling methods [J]. Behaviour, 49: 227-265.

Baker AEM. 1981. Gene flow in house mice: Behavior in a population cage [J]. Behavioral Ecology and Sociobiology, 8: 83-90.

Bulger JB. 1993. Dominance rank and access to estrous females in male savanna baboons [J]. Behaviour, 127: 67-103.

Chen R, Li JH, Zhu Y, Xia DP. 2008. Seasonal changes in aggressive behavior and fecal testosterone level in male Tibetan macaques Macaca thibitana at Huangshan, China [J]. Acta Zoologica Sinica, 54(3): 393-398. [陈 燃, 李进华, 朱 勇, 夏东坡. 2008. 雄性黄山 短尾猴攻击行为和粪便睪酮水平季节性变化. 动物学报, 54(3): 393-398.]

Clutton-Brock TH, Parker GA. 1992. Potential reproductive rates and the operation of sexual selection [J]. Quarterly Review of Biology, 67: 437-455.

Emlen ST, Oring LW. 1977. Ecology, sexual selection, and the evolution of mating systems [J]. Science, 197: 215-223.

Fred BB. 1995. Female cooperation, consortship maintenance, and male mating success in savanna baboons [J]. Animal Behaviour, 50: 137-149.

Grant JWA, Bryant MJ, Soos CE. 1995. Operational sex ratio, mediated by
释了为什么 $\mathrm{YA}_{2}$ 群经常大范围迁移, 而 $\mathrm{YA}_{1}$ 群相对稳 定的现象。因此, 对成年雄性黄山短尾猴有效性比 压力模型的研究, 可以发现雄雌有效性比过高造成 的交配竞争激烈会给黄山短尾猴社群的稳定性带 来危害。

但两群中的低顺位成年雄性在繁殖行为上无 显著差异, $\mathrm{YA}_{1}$ 群低顺位成年雄性在交配期内未观 察到有主动攻击行为的发生。这说明黄山短尾猴对 顺位具有认知能力 $(\mathrm{Li}, 2000)$, 有效性比不同的两 群低顺位个体繁殖行为发生频次均很低, 低顺位个 体也不会对高顺位个体发动攻击行为 (Chen et al, 2008), 因此我们认为成年雄性的繁殖行为和攻击 行为不仅受到有效性比压力的影响, 而且还受到包 括顺位在内的多方面因素的影响。此外, Fred (1995) 对草原狒狒 (Papio cynocephalus) 的研究发现, 雄 性个体的交配机会会随着有效性比中能够交配雌 性数量的下降而减少, 但其射精交配的机会却与有 效性比无关 (Fred, 1995)。本研究中, 由于野外观 察条件的限制, 没能辨别交配行为是否射精, 故关 于有效性比对射精交配的影响有待进一步研究。

致谢: 黄山野生猴谷管理中心谢升飞、谢玉峰 和其他工作人员和房东程新明全家对野外工作给 予了支持和帮助, 美国中部华盛顿大学 Megan Matheson 教授审阅了英文摘要，在此一并致谢!

synchrony of female arrival, alters the variance of male mating success in Japanese medaka [J]. Animal Behaviour, 49: 367-375.

Gwynne DT. 1991. Sexual competition among females: What causes courtship-role reversal [J]. Trends in Ecology and Evolution, 6: 118-121.

Jia ZY, Jiang ZG, Wang ZW. 2000. Observation on the behaviors of Masked palm civet in reproductive season [J]. Acta Theriologica Sinica, 20(2): 108-115. [贾志云, 蒋志刚, 王祖望. 2000. 果子狸繁殖期行为的观 察. 兽类学报, 20(2): 108-115.]

Jirotkul M. 1999. Operational sex ratio influences female preference and male-male competition in guppies [J]. Animal Behaviour, 58: 287-294.

Klug H, Kontax CI, Annis S, Vasudevan N. 2008. Operational sex ratio affects nest tending and aggression in male flagfish Jordanella floridae Goode \& Bean [J]. Journal of Fish Biology, 72: 1295-1305.

Kvarnemo C, Forsgren E, Magnhagen C. 1995. Effects of sex ratio on intraand inter-sexual behaviour in sand gobies [J]. Animal Behaviour, 50: 1455-1461.

Li JH, Wang QS, Li M. 1995. Studies on the population ecology of Tibetan monkeys (Macaca thibetana) III. Age structure and life table of Tibetan monkeys [J]. Acta Theriologica Sinica, 15(1): 31-35. [李进华, 王岐山, 李 明. 1995. 短尾猴种群生态学研究 III. 年龄结构和生命表. 兽 
类学报, 15(1): 31-35.]

Li JH, Wang QS, Han DM. 1996. Fission in a free-ranging Tibetan macaque troop at Huangshan Mountains, China [J]. Chinese Science Bulletin, 41: 1377-1381.

Li JH. 1999. The Tibetan Macaque Society: A Field Study [M]. Hefei: Anhui University Press. [李进华. 1999. 野生短尾猴的社会. 合肥: 安徽大 学出版社.]

Li JH. 2000. Recognition of rank relationship by Tibetan Monkeys [J]. Journal of Anhui University (Natural Science Edition), 24(3): 116-120. [李进华. 2000. 短尾猴对顺位关系的认知. 安徽大学学报(自然科学 版), 24(3): 116-120.]

Li JH, Yin HB, Wang QS. 2005. Seasonality of reproduction and sexual activity in female Tibetan macaques Macaca thibetana at Huangshan, China [J]. Acta Zoologica Sinica, 51(3): 365-375.

Li JH, Yin HB, Zhou LZ. 2007. Non-reproductive copulation behavior among Tibetan macaques Macaca thibetana at Huangshan, China [J]. Primates, 48: 64-72.

Mark AC, Lewis WO. 1988. Sex ratios and intrasexual competition for mates in a sex-role reversed shorebird, Wilson's phalarope (Phalaropus tricolor) [J]. Behavioral Ecology and Sociobiology, 22: 165-173.

Martin R, Pavel J, Carl S. 2004. Male-male interference competition decreases spawning rate in the European bitterling (Rhodeus sericeus) [J]. Behavioral Ecology and Sociobiology, 56: 34-41.

Michael H, Katrin B, Ulrike M, Dana P, John D, Keith H. 2007. Female ovarian cycle phase affects the timing of male sexual activity in free-ranging Barbary macaques (Macaca sylvanus) of Gibraltar [J]. American Journal of Primatology, 69: 1-15.

Mitani, John C. 1990. Experimental Field Studies of Asian Ape Social Systems [J]. International Journal of Primatology, 11(2): 103-126.

Norusis M. 2005. SPSS 13.0 Advanced Statistical Procedures Companion [M]. Upper Saddle River, NJ: Prentice Hall.

Newsome AE. 1969. A population study of house-mice permanently inhabiting a reed-bed in south Australia [J]. Journal of Animal Ecology, 38: $361-377$.
Patrick SF, Jean-Francois LG. 2008. Operational sex ratio, sexual conflict and the intensity of sexual selection [J]. Ecology Letters, 11: 432-439.

Paul A. 1997. Breeding seasonality affects the association between dominance and reproductive success in non-human male primates [J]. Folia Primatol, 68: 344-349.

Quinn TP, Adkison MD, Ward MB. 1996. Behavioral tactics of male sockeye salmon (Oncorhynchus nerka) under varying operational sex ratios [J]. Ethology, 102: 304-322.

Sun RY. 2001. The Principles of Animal Ecology. 3rd ed [M]. Beijing: Beijing Normal University Press. [孙儒泳. 2001. 动物生态学原理(第 三版). 北京: 北京师范大学出版社.]

Takahashi H. 2001. Influence of fluctuation in the operational sex ratio to mating of troop and non-troop male Japanese macaques for four years on Kinkazan Island [J]. Japan Primates, 42: 183-191.

Takahashi H. 2002. Female reproductive parameters and fruit availability: Factors determining onset of estrus in Japanese macaques [J]. American Journal of Primatology, 57: 141-153.

Takahashi H. 2004. Do Males have a better chance of mating when the number of estrous females is equal to or greater than the males' ordinal rank? testing the hypothesis in Japanese macaques [J]. American Journal of Primatology, 63: 95-102.

Valerie JD, Julian TA, John DR. 1999. The effects of sex ratio on sexual competition in the European lobster [J]. Animal Behaviour, 58: 973-981.

Zhang JJ, Shi DZ. 2006. Social behavior of male Brandt's vole under different group conditions [J]. Acta Theriologica Sinica, 26(2): 159-163. [张建军, 施大钊. 2006. 不同社群条件下雄性布氏田鼠的 行为. 兽类学报, 26(2): 159-163.]

Zhu Y, Li JH, Xia DP, Chen R, Sun BH. 2008. Inbreeding avoidance by female Tibentan macaques Macaca thibetana at Huangshan,China [J]. Acta Zoologica Sinica, 54(2): 183-190. [朱勇, 李进华, 夏东坡, 陈 燃, 孙丙华. 2008. 雌性黄山短尾猴回避近亲交配. 动物学报, 54(2): 183-190.] 